\title{
Stochastic Model Predictive Control based on Gaussian Processes applied to Drinking Water Networks
}

\author{
Ye Wang, Carlos Ocampo-Martinez, and Vicenç Puig
}

\begin{abstract}
This paper focuses on developing a stochastic model predictive control (MPC) strategy based on Gaussian Processes (GPs) for propagating system disturbances in a receding horizon way. Using a probabilistic system representation, the state trajectories considering the influence of disturbances can be obtained through the uncertainty propagation by using GPs. This fact allows obtaining the confidence intervals for state evolutions over the MPC prediction horizon that are included into the MPC objective function and constraints. The feasibility of the proposed MPC strategy considering the incorporated results of disturbance forecasting is also discussed. Simulation results obtained from the application of the proposed approach to the Barcelona drinking water network (DWN) taking real demand data into account are presented. The comparison with the well-known certainty-equivalent MPC shows the effectiveness of the proposed stochastic MPC approach.
\end{abstract}

\section{Index Terms}

Stochastic Model Predictive Control, Gaussian Processes, Disturbance Forecasting, Drinking Water Networks.

\section{INTRODUCTION}

System disturbances and (parametric/structural) uncertainties can have a deep influence on the performance of a control system. The inclusion of robustness in model predictive control (MPC) is a well-known research field, which deals with system disturbances and uncertainties [1], [2]. Considering the way on how both disturbances and uncertainties are modelled, robust MPC is divided into deterministic MPC (DMPC) and stochastic MPC (SMPC). In the former case, disturbances and uncertainties are represented by bounded sets while for the latter they are represented by their probability distributions.

MPC with Gaussian Process (GP) models was firstly introduced in [3], where it is proposed a new way of considering uncertainty into the MPC design. Likewise, predictive control together with GP have also been considered for addressing

Y. Wang, C. Ocampo-Martinez and V. Puig are with Advanced Control System (SAC) Research Group at Institut de Robòtica i Informàtica Industrial, CSIC-UPC, Universitat Politècnica de Catalunya - BarcelonaTech, Llorens i Artigas 4-6. 08028 Barcelona, Spain. (e-mail: \{ywang,cocampo,vpuig\}@ iri.upc.edu). 
the fault-tolerant problem [4]. Generally speaking, the GP approach is able to provide a new way of including the system disturbances by means of probability theory in the SMPC framework. GP regression models have been applied to many different real cases such as electricity forecasting [5], [6], disturbance forecasting in greenhouse temperature control systems [7], among other fields. There are some other methodologies for electricity forecasting that have been discussed in the past decades, such as artificial neural networks [8], [9]. These algorithms have also been employed for the water demand forecasting [10], [11]. The superiority of GP regression comes from the use of the Bayesian Inference Theory, which is able to update in real time the parameters of a GP regression model adapted to accumulated data [12].

In terms of the MPC strategy, the system model is used to find an optimal control action along a prediction horizon according to the given performance objectives and physical/operational constraints. Thus, a modeling approach should be needed, which is not only able to consider system dynamics but also the dynamic effect of exogenous disturbances. Hence, a proper disturbance forecasting approach should be used to produce reliable short-term forecasting results. In this framework, some forecasting approaches have been discussed in [13], [14]. After applying a disturbance forecasting approach, the results will be incorporated into the optimal control input computation. On the other hand, the optimization problem from an MPC controller could be infeasible in some particular time instant in a closed-loop control system since the nature of its disturbances might be stochastic. Furthermore, forecasting results have a wide variance interval and they are included into the open-loop optimization along the MPC prediction horizon. Then, a method is proposed to address the infeasibility that could occur when the system state trajectories evolve outside their operational limitations when driven by the disturbance forecasting results.

The main contribution of this paper relies on the design of a SMPC controller based on GP taking infeasibility analysis into account when using the disturbance forecasting results obtained from the approach proposed in [14], which is called in the sequel $D S H W-G P$. This approach combines the double-seasonal Holt-Winters (DSHW) method and GP regression algorithms in order to propagate the disturbance forecast uncertainty into the system states. DSHWGP is a mixed methodology that incorporates advantages from both the DSHW and GP algorithms. Unlike traditional forecasting approaches, e.g., [15], [16], the DSHW-GP algorithm is useful since it allows to model the uncertain part of the disturbance forecast in the GP framework. A case study based on the Barcelona drinking water network (DWN) has been considered using real demand data, where the aforementioned SMPC strategy is applied and assessed by means of some given key performance indicators (KPIs).

The remainder of this paper is structured as follows. In Section II, the problem formulation is presented. The SMPC strategy based on the GP approach is presented in Section III. In Section IV, the application of the considered approach to Barcelona DWN is described, while simulation results are shown in Section V. Finally, the main conclusions are drawn in Section VI. 


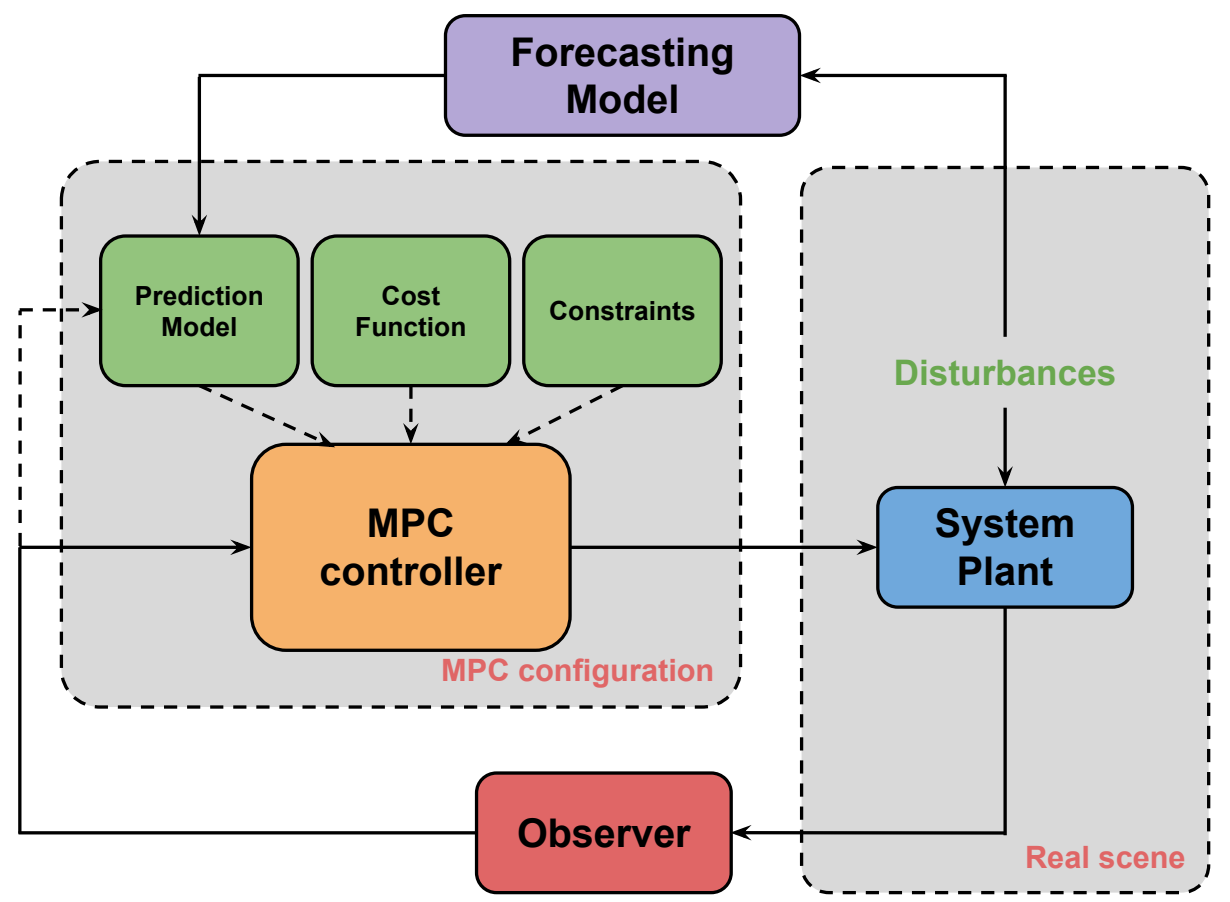

Figure 1. Components of a SMPC closed-loop topology

\section{Problem Statement}

\section{A. Control-oriented Model}

Consider that the system to be controlled can be described by means of a discrete-time control-oriented model

$$
\mathbf{x}_{k+1}=\mathbf{A} \mathbf{x}_{k}+\mathbf{B} \mathbf{u}_{k}+\mathbf{d}_{k}
$$

where $\mathbf{x} \in \mathbb{R}^{n}$ and $\mathbf{u} \in \mathbb{R}^{m}$ denote system state and control input vectors, respectively. Moreover, $\mathbf{A}, \mathbf{B}$ are the system matrices of suitable dimensions, $\mathbf{d} \in \mathbb{R}^{q}$ represents the vector of system disturbances and $k$ is the discrete time.

The components of a SMPC-based closed loop are shown in Figure 1. There, the system is affected by the influence of external disturbances, which should be properly forecasted facing their inclusion in the prediction model used by the SMPC controller. Notice that the loop is closed by using an observer, which allows to consider an output-feedback scheme. This paper assumes that the observer model is not affected by the disturbances.

\section{B. Disturbance Forecasting Approach}

According to (1), system states are influenced by the exogenous disturbances. As stated before, the disturbance forecasting approach considered in this paper is the DSHW-GP algorithm, a probabilistic approach reported in [14]. 
The forecasting results are not a set of deterministic values but a set of probabilities denoted by

$$
\mathbf{d}_{k} \sim \mathcal{N}\left(\overline{\mathbf{d}}_{k}, \Sigma_{\mathbf{d}_{k}}\right)
$$

where $\mathcal{N}$ is the normal (or Gaussian) distribution in the probability theory, $\overline{\mathbf{d}}_{k}$ and $\Sigma_{\mathbf{d}_{k}}$ correspond to the mean and variance disturbance estimates, which are used later on for the SMPC controller design.

\section{Generalized SMPC}

From (2), the prediction model related to the SMPC design is written in a probabilistic way as

$$
\mathbf{x}_{k} \sim \mathcal{N}\left(\overline{\mathbf{x}}_{k}, \Sigma_{\mathbf{x}_{k}}\right)
$$

where $\overline{\mathbf{x}}_{k}, \Sigma_{\mathbf{x}_{k}}$ are the mean and the variance of the system states $\mathbf{x}_{k}$, respectively. This paper addresses the optimization problem behind the SMPC design with two probabilistic models: $(i)$ the probabilistic representation of the state-space model of the system and (ii) the forecasting model of the disturbances. These models are used to find a sequence of control actions over a given prediction horizon $H_{p} \in \mathbb{N}_{+}$. The receding horizon approach is used for selecting the first control action of the optimal sequence at each time instant. In general, a basic SMPC controller is formulated by solving the multi-objective finite-horizon optimization problem (FHOP) in Problem 1.

Problem 1 (Generalized FHOP).

$$
\min _{\mathbf{u}_{k}^{*}} \triangleq \mathbb{E}\left[\sum_{n=1}^{\Gamma} \sum_{i=0}^{H_{p}-1} \lambda_{n} \ell_{n}\left(\mathbf{x}_{k+i+1 \mid k}, \mathbf{u}_{k+i \mid k}, \mathbf{d}_{k+i \mid k}\right)\right],
$$

subject to

$$
\begin{aligned}
& \mathbf{x}_{k+i+1 \mid k} \sim \mathcal{N}\left(\overline{\mathbf{x}}_{k+i+1 \mid k}, \Sigma_{\mathbf{x}_{k+i+1 \mid k}}\right), \\
& \mathbf{d}_{k+i \mid k} \sim \mathcal{N}\left(\overline{\mathbf{d}}_{k+i \mid k}, \Sigma_{\mathbf{d}_{k+i \mid k}}\right) \\
& \mathbf{u}_{\min } \leq \mathbf{u}_{k+i \mid k} \leq \mathbf{u}_{\max } \\
& \mathbf{x}_{\min } \leq \mathbf{x}_{k+i+1 \mid k} \leq \mathbf{x}_{\max } \\
& \left(\mathbf{x}_{k \mid k}, \mathbf{d}_{k \mid k}\right)=\left(\mathbf{x}_{k}, \mathbf{d}_{k}\right)
\end{aligned}
$$

where $\Gamma \in \mathbb{N}_{+}$is the total number of control objectives $\ell_{n}$ that conform the total objective function using a weighting approach where $\lambda_{n}$ are the weights, $\mathbb{E}$ is the expectation operator, together with mean parameters $\overline{\mathbf{x}}_{k+i+1 \mid k}, \bar{d}_{k+i \mid k}$ and variance parameters $\Sigma_{\mathbf{x}_{k+i+1 \mid k}}, \Sigma_{\mathbf{d}_{k+i+1 \mid k}}$. Moreover, $\mathbf{u}_{\min }, \mathbf{u}_{\max }$ are two vectors with suitable dimensions defining the limitations of the input variables, respectively, while $\mathbf{x}_{\min }, \mathbf{x}_{\max }$ are the limitations of the system states, respectively. 
Remark 1 (Feasibility). Given the stochastic nature of future disturbances (2), the prediction model (1) involves exogenous (additive) uncertainty. Thus, the satisfaction of constraints (4e) for a given control input cannot be ensured with the explicit consideration of uncertainty. This means that, even if the predictive controller finds a feasible solution to achieve the operational goals ignoring uncertainty, there is a given probability that real outputs may violate constraints (4e). To preserve feasibility, the proposed GP-MPC controller will soften the constraints (4e) by including a slack variable such that those constraints are satisfied when considering the worst-case state evolution along the prediction horizon. This mechanism will also allow to establish a trade-off between feasibility and performance.

\section{Stochastic Model Predictive Control based on Gaussian Processes}

In this section, an SMPC controller is generally designed also based on the GP approach as used in the disturbance forecasting. The GP is adopted to propagate system disturbances into the system states over the MPC prediction horizon. Hence, not only the disturbance forecasting model but also the system state model are both characterized by means of a GP model. Hence, the SMPC controller is named GP-MPC in the remaining of this paper. Furthermore, it is necessary to discuss the feasibility of the GP-MPC.

\section{A. Probabilistic Prediction Model}

By deploying the DSHW-GP approach in [14], the forecasting disturbances over $H_{p}$ are obtained as

$$
\mathbf{d}_{k+i} \sim \mathcal{G} \mathcal{P}\left(\overline{\mathbf{d}}_{k+i}, \Sigma_{\mathbf{d}_{k+i}}\right), i=0,1,2, \ldots, H_{p}-1
$$

where $\overline{\mathbf{d}}_{k+i}$ is the mean estimation and $\Sigma_{\mathbf{d}_{k+i}}$ is the variance estimation, both at time instant $k+i$. $\mathcal{G P}$ is the GP model defined in [14].

Under a probabilistic representation, the prediction model can be written as $p\left(\mathbf{x}_{i+1}\right) \sim \mathcal{N}\left(\overline{\mathbf{x}}_{k+1}, \Sigma_{\mathbf{x}_{k+1}}\right)$ and the state transition probability can be approximated by

$$
\left(\mathbf{x}_{k}, \mathbf{x}_{k+1} \mid \overline{\mathbf{x}}_{k}, \Sigma_{\mathbf{x}_{k}}\right) \sim \mathcal{N}\left(\left[\begin{array}{c}
\overline{\mathbf{x}}_{k} \\
\overline{\mathbf{x}}_{k+1}
\end{array}\right],\left[\begin{array}{cc}
\Sigma_{\mathbf{x}_{k}} & \Sigma_{\mathbf{x}_{k}, \mathbf{x}_{k+1}} \\
\Sigma_{\mathbf{x}_{k}, \mathbf{x}_{k+1}}^{T} & \Sigma_{\mathbf{x}_{k+1}}
\end{array}\right]\right),
$$

where $\overline{\mathbf{x}}_{k}$ and $\overline{\mathbf{x}}_{k+1}$ represent the mean values of the states at two consecutive steps, respectively, while $\Sigma_{\mathbf{x}_{k}}$ and $\Sigma_{\mathbf{x}_{k+1}}$ represent the corresponding variance matrices of states. Moreover, $\Sigma_{\mathbf{x}_{k}, \mathbf{x}_{k+1}}$ denotes the covariance matrix of the states at time instant $k$ and $k+1$, respectively. Since the system model in (1) is linear, then (6) can be simplified as

$$
\left[\begin{array}{c}
\mathbf{x}_{k} \\
\mathbf{x}_{k+1}
\end{array}\right] \sim \mathcal{N}\left(\left[\begin{array}{c}
\overline{\mathbf{x}}_{k} \\
\overline{\mathbf{x}}_{k+1}
\end{array}\right],\left[\begin{array}{cc}
\Sigma_{\mathbf{x}_{k}} & \Sigma_{\mathbf{x}_{k}, \mathbf{x}_{k+1}} \\
\Sigma_{\mathbf{x}_{k}, \mathbf{x}_{k+1}}^{T} & \mathbf{A} \Sigma_{\mathbf{x}_{k}} \mathbf{A}^{T}+\Sigma_{\mathbf{d}_{k}}
\end{array}\right]\right)
$$

Assumption 1. Each state is time independent. The state uncertainty corresponding to the time instant $k+1$ is propagated by using the state value at $k$. 
According to Assumption 1, the mean and variance of the system states are written as

$$
\begin{aligned}
\overline{\mathbf{x}}_{k+1} & =\mathbf{A} \overline{\mathbf{x}}_{k}+\mathbf{B} \mathbf{u}_{k}+\overline{\mathbf{d}}_{k}, \\
\Sigma_{\mathbf{x}_{k+1}} & =\mathbf{A} \Sigma_{\mathbf{x}_{k}} \mathbf{A}^{T}+\Sigma_{\mathbf{d}_{k}} .
\end{aligned}
$$

In order to transform the general constraints for all the system states into individual constraints, it is necessary to find a probabilistic-based procedure. By means of a risk term $\alpha$, the joint chance constraint of the system state is drawn as [17]

$$
\operatorname{Pr}\left(\mathbf{x}_{\min } \leq \mathbf{x}_{k} \leq \mathbf{x}_{\max }\right)=1-\alpha
$$

where $\alpha$ is the risk in terms of the confidence level $(1-\alpha)$ and $\operatorname{Pr}$ denotes the probability. Due to further state evolution considered in the receding horizon strategy, the expected global risk can be allocated into each state and each step over $H_{p}$. Therefore, (9) can be rewritten for each state as

$$
\begin{gathered}
\operatorname{Pr}\left(\mathbf{x}_{\min , \mathrm{i}} \leq \mathbf{x}_{k+j, i} \leq \mathbf{x}_{\max , \mathrm{i}}\right)=1-\alpha_{i, j}, \\
\sum_{i=1}^{n_{x}} \sum_{j=1}^{H_{p}} \alpha_{i, j} \leq \alpha_{\text {global }}, \\
0 \leq \alpha_{i, j} \leq 1,
\end{gathered}
$$

where $i$ and $j$ denote indices for state and prediction step respectively, $\alpha_{i, j}$ is the partial risk averaged into each state and the sum of all partial risks is close to the global risk $\alpha_{\text {global }}$. In other words, the global risk can be allocated into fixed partial risks using the following expression:

$$
\alpha_{i, j}=\frac{\alpha_{\text {global }}}{n_{c}}
$$

where $n_{c}$ denotes the value used for allocating the global risk into partial risks.

\section{B. Constraints Reformulation}

According to [17], the convexity of an optimization problem with joint-chance constraints has been discussed. Hence, (10a) can be decomposed into the following expressions:

$$
\begin{aligned}
\overline{\mathbf{x}}_{j, i}+c \Sigma_{x_{j, i}}^{1 / 2} & \leq \mathbf{x}_{\max , i} \\
\overline{\mathbf{x}}_{j, i}-c \Sigma_{\mathbf{x}_{j, i}}^{1 / 2} & \geq \mathbf{x}_{\min , i},
\end{aligned}
$$

where $\overline{\mathbf{x}}_{j, i}+c \Sigma_{x_{j, i}}^{1 / 2}$ is the upper bound of the $i$-th state at the time instant $j$, which should be lower than $\mathbf{x}_{\max , i}$ while $\overline{\mathbf{x}}_{j, i}-c \Sigma_{\mathbf{x}_{j, i}}^{1 / 2}$ is the lower bound of the $i$-th state at the time instant $j$, which should be greater than $\mathbf{x}_{\min , i}$. Hence, 




Figure 2. States propagation with uncertainty along $H_{p}$

the worst-case state evolutions are reflected into the MPC constraints. The critical value $c$ in (12) can be computed by means of the inverse standard probability density function $\Phi^{-1}$ as

$$
\begin{aligned}
c & =\Phi^{-1}\left(1-\alpha_{i, j}\right), \\
\alpha_{i, j} & =\frac{\alpha_{\text {global }}}{2 n_{x} H_{p}} .
\end{aligned}
$$

In order to analyze the constraint feasibility for all system states, (12) can be rewritten as

$$
\mathbf{x}_{\min , i}^{\prime} \leq \overline{\mathbf{x}}_{j, i} \leq \mathbf{x}_{\max , \mathrm{i}}^{\prime} \forall i \in\left[1, n_{x}\right]
$$

where

$$
\begin{aligned}
& \mathbf{x}_{\max , i, j}^{\prime} \triangleq \mathbf{x}_{\max , i}-c \Sigma_{x_{j, i}}^{1 / 2} \\
& \mathbf{x}_{\min , i, j}^{\prime} \triangleq \mathbf{x}_{\min , i}+c \Sigma_{x_{j, i}}^{1 / 2} .
\end{aligned}
$$

The optimization problem behind the predictive controller design would be infeasible when there is overlapped areas between $\mathbf{x}_{\max , i, j}^{\prime}$ and $\mathbf{x}_{\min , i, j}^{\prime}$ over $H_{p}$, see the infeasible point in Figure 2. In this case, for time instants after that point, the optimization problem becomes infeasible. Hence, it is necessary to verify whether $\mathbf{x}_{\max , i, j}^{\prime}$ is always greater than $\mathbf{x}_{\min , i, j}^{\prime}$ over $H_{p}$ for all system states.

To avoid this situation, some solutions have been discussed, e.g., the risk allocation, see [18]. The risk can be allocated into each state and each step along $H_{p}$ through an additional optimization problem. Another solution by 
means of the terminal constraint is exploited by slacking $c$ by using a soft term $\eta$, which means that the critical value is not fixed but variable such that $c_{j}$ has to be optimized depending on the state uncertainty. If it is overestimated, the optimal soft term can be found in order to satisfy the condition: $\mathbf{x}_{\max , i, j}^{\prime}$ is always greater than $\mathbf{x}_{\min , i, j}^{\prime}$, i.e.,

$$
\begin{gathered}
\mathbf{x}_{\max , i}-\gamma \geq \mathbf{x}_{\min , i}+\gamma, \\
\gamma=\left(c-\eta_{j}\right) \Sigma_{x_{i, j+1}}^{1 / 2} \quad \forall j \in\left[1, H_{p}\right] .
\end{gathered}
$$

Meanwhile, the variance of the system states in (8b) is growing. Hence, since the state uncertainty is accumulated, the soft term is growing as well. Generally, the constraints for $c_{j}$ are defined as

$$
\begin{gathered}
c_{j}=c_{0}-\eta_{j}, \\
\eta_{j} \leq \eta_{j+1},
\end{gathered}
$$

where $c_{0}$ is the initial critical value. Similarly, $\eta_{j} \in \mathbb{R}^{n_{x} H_{p}}$ is the soft term.

\section{Objective Function}

The other key component in MPC design is the objective function, that is also named as the cost function. As pointed out, $c$ has been slacked by the soft term $\eta_{j}$ in (17a) and (17b) for the purpose of coping with an uncontrolled growing of uncertainty. The expectation for relaxing $c$ is to find a minimal value to maintain the partial risk at each step of the MPC controller computation. This is achieved by including in the MPC objective function the following term

$$
\ell_{k}^{c} \triangleq\left\|\eta_{k}\right\|_{2, \mathbf{w}_{c}}^{2}
$$

where $\mathbf{W}_{c}$ denotes the weighting factor when included in the general objective function. Note that $\|\cdot\|_{p, \mathbf{W}}$ denotes a weighted $p$-norm.

\section{GP-MPC Setting}

The constraints for states are generated with the probabilistic prediction model. The total objective function is the sum of objective functions for each operational goal with a group of weights $\lambda_{n}$, which can be chosen depending on the prioritization of the considered objectives. Therefore, the general optimization problem discussed in this paper is formulated in Problem 2.

Problem 2 (Improved GP-MPC).

$$
\min _{\mathbf{u}_{k}^{*}, \eta_{k}^{*}} \mathbb{E}\left[\sum_{n=1}^{\Gamma} \sum_{i=0}^{H_{p}-1} \lambda_{n} \ell^{n}\left(\mathbf{x}_{k+i \mid k}, \mathbf{u}_{k+i \mid k}, \mathbf{d}_{k+i \mid k}\right)\right],
$$


subject to

$$
\begin{aligned}
& \overline{\mathbf{x}}_{k+i+1 \mid k}=\mathbf{A} \overline{\mathbf{x}}_{k+i \mid k}+\mathbf{B} \mathbf{u}_{k+i \mid k}+\overline{\mathbf{d}}_{k+i \mid k}, \\
& \Sigma_{\mathbf{x}_{k+i+1 \mid k}}=A \Sigma_{\mathbf{x}_{k+i \mid k}} A^{T}+\Sigma_{\mathbf{d}_{k+i \mid k}}, \\
& \mathbf{u}_{\min } \leq \mathbf{u}_{k+i \mid k} \leq \mathbf{u}_{\max }, \\
& \overline{\mathbf{x}}_{k+i+1 \mid k}+\left(c-\eta_{k+i \mid k}\right) \Sigma_{x_{k+i+1 \mid k}}^{1 / 2} \leq \mathbf{x}_{\max }, \\
& \overline{\mathbf{x}}_{k+i+1 \mid k}-\left(c-\eta_{k+i \mid k}\right) \Sigma_{\mathbf{x}_{k+i+1 \mid k}}^{1 / 2} \geq \mathbf{x}_{\min }, \\
& \mathbf{x}_{\max }-\gamma \geq \mathbf{x}_{\min }+\gamma, \\
& \gamma=\left(c-\eta_{k+H_{p} \mid k}\right) \Sigma_{x_{k+H_{p}+1 \mid k}}^{1 / 2}, \\
& \eta_{k+i \mid k} \leq \eta_{k+i+1 \mid k}, \\
& \left(\mathbf{x}_{k \mid k}, \mathbf{d}_{k \mid k}\right)=\left(\mathbf{x}_{k}, \mathbf{d}_{k}\right)
\end{aligned}
$$

Remark 2 (Stability). As discussed in Remark 1, the feasibility of the proposed GP-MPC strategy can be guaranteed by means of the mechanisms previously proposed and discussed. Although feasibility can imply stability for the MPC strategy as discussed in [19], in order to guarantee stability the inclusion of some terminal constraints in order to enforce the system state to evolve towards an invariance set (previously designed) is additionally required, see [20], [21].

\section{Case Study: Barcelona Drinking Water Network}

\section{A. System Description}

The Barcelona DWN supplies $237.7 \mathrm{hm}^{3}$ of drinking water to approximately three million consumers every year, covered $424 \mathrm{~km}^{2}$ area. The entire network is composed of 63 storage tanks, three surface sources, seven underground sources, 79 pumps, 50 valves, 18 nodes and 88 water demands. Currently, AGBAR (Aguas de Barcelona, S. A. Company which manages the drinking water transport and distribution in Barcelona, Spain) is in charge of managing the entire network through a supervisory control system. It supplies potable water to the Metropolitan Area of Barcelona (Catalonia, Spain). The main water resources come from rivers Llobregat, Ter and Besòs, with $52 \%$, $46 \%$ and $2 \%$ of the total water supply, respectively. These sources are regulated by dams that have an overall capacity of 600 cubic hectometres.

Figure 3 shows a representative portion of the Barcelona DWN selected as the case study of this paper. This DWN part includes three water storage tanks, three pumps, three valves and four water demand sectors. The sampling time for collecting water demands from the consume sectors is 1 hour. The mathematical model case study as well as other mathematical features can be found in [22].The parameters of the model have been adjusted by using real data provided 


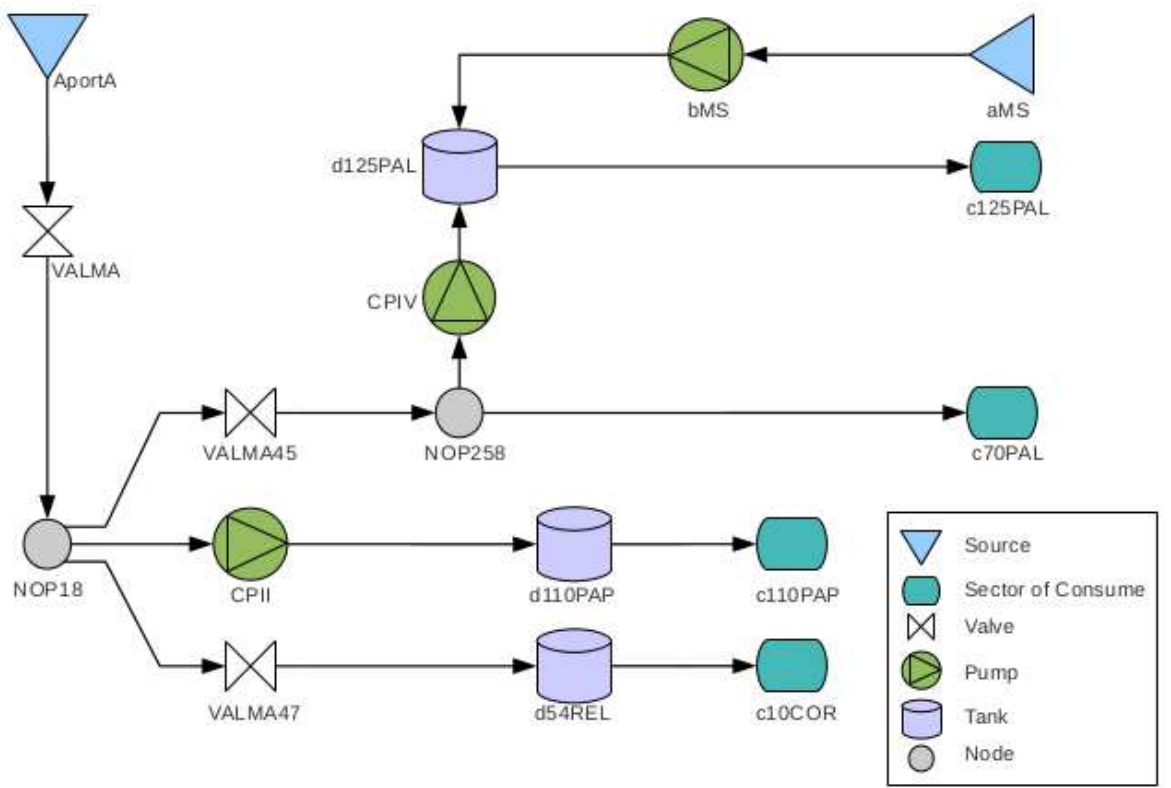

Figure 3. The three-tank system topology

by the DWN management company.

\section{B. Control-oriented Model}

The control-oriented model of DWN is described by the following set of linear discrete-time difference-algebraic equations for all time instant $k \in \mathbb{N}[17]$ :

$$
\begin{aligned}
\mathbf{x}_{k+1} & =\mathbf{A} \mathbf{x}_{k}+\mathbf{B} \mathbf{u}_{k}+\mathbf{B}_{d} \mathbf{d}_{k} \\
0 & =\mathbf{E}_{u} \mathbf{u}_{k}+\mathbf{E}_{d} \mathbf{d}_{k}
\end{aligned}
$$

where $\mathbf{x}_{k}, \mathbf{u}_{k}, \mathbf{d}_{k}$ denote the state vector, the manipulated flows through actuators and the vector of demanded flow as additive measured disturbances, respectively. Pumps and valves are considered as the actuators. Moreover, (20a) describes the dynamics of storage tanks while (20b) presents the static relations within the DWN at network nodes. Matrices $\mathbf{A}, \mathbf{B}, \mathbf{B}_{d}, \mathbf{E}_{u}$ and $\mathbf{E}_{d}$ are obtained from the network topology (see [22], for the numerical values of the part of the network considered in this paper).

For the purpose of incorporating disturbance forecasting results into system states by using the GP again, the standard linear control-oriented model as (1) is required. A compact form of (20) can be written as

$$
\mathbf{x}_{k+1}=\mathbf{A} \mathbf{x}_{k}+\tilde{\mathbf{B}} \tilde{\mathbf{u}}_{k}+\tilde{\mathbf{B}}_{p} \mathbf{d}_{k}
$$


where $\tilde{\mathbf{u}}_{k}$ is the reduced vector control inputs expressed as

$$
\mathbf{u}_{k}=\tilde{\mathbf{P}} \tilde{\mathbf{M}}_{1} \tilde{\mathbf{u}}_{k}+\tilde{\mathbf{M}}_{2} \mathbf{d}_{k}
$$

with $\tilde{\mathbf{B}}, \tilde{\mathbf{B}_{p}}, \tilde{\mathbf{P}}, \tilde{\mathbf{M}}_{1}, \tilde{\mathbf{M}}_{2}$ being resultant matrices of suitable dimensions. The detailed transformation procedure can be found in [17].

Since the DWN model is linear, the probabilistic prediction model of the DWN can be calculated in the same way as aforementioned from (6) to (8) such that the model is finally written as

$$
\begin{gathered}
\overline{\mathbf{x}}_{k+1}=\mathbf{A} \overline{\mathbf{x}}_{k}+\widetilde{\mathbf{B}} \mathbf{u}_{k}+\widetilde{\mathbf{B}}_{p} \overline{\mathbf{d}}_{k}, \\
\Sigma_{\mathbf{x}_{k+1}}=\mathbf{A} \Sigma_{\mathbf{x}_{k}} \mathbf{A}^{T}+\widetilde{\mathbf{B}}_{p} \Sigma_{\mathbf{d}_{k}} \widetilde{\mathbf{B}}_{p}^{T} .
\end{gathered}
$$

\section{Management Criteria}

The main operational goals in the management of the DWN are economic, safety, smoothness, and are respectively stated as follows [17], [23]:

1) Provide a reliable water supply minimising both water production and transport costs.

2) Guarantee the availability of enough water in each storage tank to satisfy its underlying stochastic demand.

3) Operate the DWN under smooth control actions.

\section{Physical and Operational Constraints}

Since some manipulated variables $\mathbf{u}_{k}$ have relationships with some water demands in (22), the constraint for reduced manipulated variable $\widetilde{\mathbf{u}}_{k}$ should maintain the constraint for the full manipulated variables $u_{k}$. Hence,

$$
\mathbf{u}_{\min } \leq \tilde{\mathbf{P}} \tilde{\mathbf{M}}_{1} \tilde{\mathbf{u}}_{k}+\tilde{\mathbf{M}}_{2} \mathbf{d}_{k} \leq \mathbf{u}_{\max }
$$

where $\mathbf{u}_{\min }$ and $\mathbf{u}_{\max }$ are the physical limitations of the manipulated variables $\mathbf{u}_{k}$.

On the other hand, the system states are also bounded according to the following expressions:

$$
\begin{gathered}
\mathbf{x}_{\min } \leq \mathbf{x}_{k} \leq \mathbf{x}_{\max } \\
\mathbf{x}_{k} \geq \vartheta_{\text {net }, k},
\end{gathered}
$$

where $\mathbf{x}_{\min }$ and $\mathbf{x}_{\max }$ are limitations of water storage tanks. Moreover, $\vartheta_{\text {net }, k}$ represents net demands at time instant $k$, which can be decomposed into two parts, the endogenous demands from neighbouring tanks or nodes $\mathbf{B}_{e} \mathbf{u}_{k}$ and the exogenous demands from consumer sectors $\mathbf{B}_{p} \mathbf{d}_{k}$. Hence, $\vartheta_{\text {net }, k}$ can be computed as

$$
\vartheta_{\text {net }, k}=\left|\mathbf{B}_{e} \mathbf{u}_{k}+\mathbf{B}_{p} \mathbf{d}_{k}\right| .
$$


As discussed in Section III, (25a) can be reformulated as

$$
\begin{aligned}
& \overline{\mathbf{x}}_{k+1}+\left(c-\eta_{k}\right) \Sigma_{x_{k+1}}^{1 / 2} \leq \mathbf{x}_{\max } \\
& \overline{\mathbf{x}}_{k+1}-\left(c-\eta_{k}\right) \Sigma_{\mathbf{x}_{k+1}}^{1 / 2} \geq \mathbf{x}_{\min } .
\end{aligned}
$$

\section{E. Objective Functions}

According to the safety criteria, (25b) can be set as a soft constraint in the following way:

$$
\overline{\mathbf{x}}_{k} \geq \vartheta_{\text {net }, k}-\xi_{k} \geq \mathbf{0} \quad \forall k
$$

where $\xi_{k} \in \mathbb{R}^{n_{x}}$ represents the amount of volume in $\mathrm{m}^{3}$ that goes down from the desired safety thresholds. Then, this approach introduces a new performance indicator to be minimised, defined as

$$
\ell_{k}^{s} \triangleq\left\|\xi_{k}\right\|_{2, \mathbf{w}_{x}}^{2}
$$

where $\mathbf{W}_{x}$ is the weight matrix allocated for realizing the soft constraint.

Regarding the first and third management criterion, they can be mathematically formulated as follows:

$$
\begin{aligned}
\ell_{k}^{e} & \triangleq\left\|\left(\alpha_{1}+\alpha_{2, k}\right)^{T} \mathbf{u}_{k}\right\|_{1, \mathbf{w}_{e}}, \\
\ell_{k}^{\Delta u} & \triangleq\left\|\Delta \mathbf{u}_{k}\right\|_{2, \mathbf{w}_{u}}^{2},
\end{aligned}
$$

where $\ell_{k}^{e} \in \mathbb{R}$ represents the network operation economic cost taking into account water production cost $\alpha_{1} \in \mathbb{R}^{n_{u}}$ and water pumping cost $\alpha_{2} \in \mathbb{R}^{n_{u}}$, which changes every time instant $k$ according to the variable electric tariff; $\ell_{k}^{\Delta u} \in \mathbb{R}$ represents the penalisation of control signal variations $\Delta \mathbf{u}_{k} \triangleq \mathbf{u}_{k}-\mathbf{u}_{k-1}$, to extend actuators life and assure a smooth operation; $\mathbf{W}_{e}, \mathbf{W}_{u}$ are diagonal matrices that weight each decision variable in the corresponding control objective.

Together with the objective function in (18), the total objective function of the FHOP considered for fulfilling all the management criterion is the sum of the mentioned four objective functions (18), (29), (30a) and (30b). Moreover, the control objectives are prioritised through weights $\lambda_{1}, \lambda_{2}, \lambda_{3}, \lambda_{4}$ and aggregated in the performance index. Generally speaking, the objective function of the GP-MPC optimization problem can be reformulated as

$$
\mathcal{L}_{k}=\lambda_{1} \ell_{k}^{e}+\lambda_{2} \ell_{k}^{s}+\lambda_{3} \ell_{k}^{\Delta u}+\lambda_{4} \ell_{k}^{c}
$$

\section{F. Optimization Problem Formulation}

The predication horizon $H_{p}$ is equal to 24 with a control sampling time of one hour. These values are selected taking into account the seasonality of the demand and the dynamics of the network. The GP-MPC law for the DWN consists in solving a FHOP given in Problem 3. 
Problem 3 (GP-MPC for DWN).

$$
\min _{\mathbf{u}_{k}^{*}, \eta_{k}^{*}, \xi_{k}^{*}} \sum_{i=0}^{H_{p}-1} \mathcal{L}_{k+i \mid k},
$$

subject to

$$
\begin{aligned}
& \overline{\mathbf{x}}_{k+i+1 \mid k}=\mathbf{A} \overline{\mathbf{x}}_{k+i \mid k}+\widetilde{\mathbf{B}} \widetilde{\mathbf{u}}_{k+i \mid k}+\widetilde{\mathbf{B}}_{p} \overline{\mathbf{d}}_{k+i \mid k}, \\
& \Sigma_{\mathbf{x}_{k+i+1 \mid k}}=A \Sigma_{\mathbf{x}_{k+i \mid k}} A^{T}+\widetilde{\mathbf{B}}_{p} \Sigma_{\mathbf{d}_{k+i \mid k}} \widetilde{\mathbf{B}}_{p}^{T}, \\
& \mathbf{u}_{\min } \leq \tilde{\mathbf{P}} \tilde{\mathbf{M}}_{1} \tilde{\mathbf{u}}_{k+i \mid k}+\tilde{\mathbf{M}}_{2} \mathbf{d}_{k} \leq \mathbf{u}_{\max }, \\
& \overline{\mathbf{x}}_{k+i+1 \mid k}+\left(c-\eta_{k+i \mid k}\right) \Sigma_{x_{k+i+1 \mid k}}^{1 / 2} \leq \mathbf{x}_{\max }, \\
& \overline{\mathbf{x}}_{k+i+1 \mid k}-\left(c-\eta_{k+i \mid k}\right) \Sigma_{\mathbf{x}_{k+i+1 \mid k}}^{1 / 2} \geq \mathbf{x}_{\min }, \\
& \mathbf{x}_{\max }-\gamma \geq \mathbf{x}_{\min }+\gamma, \\
& \gamma=\left(c-\eta_{k+H_{p} \mid k}\right) \Sigma_{x_{k+H}+2}^{1 / 2}, \\
& \eta_{k+i \mid k} \leq \eta_{k+i+1 \mid k}, \\
& \overline{\mathbf{x}}_{k+i+1 \mid k} \geq \vartheta_{\mathrm{net}, k+i+1 \mid k}-\xi_{k+i \mid k} \geq 0 \\
& \left(\mathbf{x}_{k \mid k}, \mathbf{d}_{k \mid k}\right)=\left(\mathbf{x}_{k}, \mathbf{d}_{k}\right) .
\end{aligned}
$$

\section{Simulation Results}

\section{A. Key Performance Indicators}

For the sake of comparing the performance of the proposed GP-MPC strategy with different risk settings, the following key performance indicators (KPIs) are utilized:

$$
\begin{aligned}
& K P I_{E} \triangleq \frac{1}{n_{s}} \sum_{k=1}^{n_{s}}\left|\left(\alpha_{1}+\alpha_{2, k}\right)^{T} \mathbf{u}_{k}\right| \\
& K P I_{S} \triangleq \sum_{k=1}^{n_{s}} \sum_{i=1}^{n_{x}} \max \left\{0,\left(\vartheta_{\operatorname{net}(i), k}-\mathbf{x}_{(i), k}\right)\right\} \\
& K P I_{M} \triangleq \frac{1}{n_{s}} \sum_{k=1}^{n_{s}} \sum_{i=1}^{n_{x}}\left(\mathbf{x}_{(i), k}-\vartheta_{\operatorname{net}(i), k}\right) \\
& K P I_{\Delta U} \triangleq \frac{1}{n_{s}} \sum_{k=1}^{n_{s}} \sum_{i=1}^{n_{u}}\left(\Delta \mathbf{u}_{(i), k}\right)^{2}
\end{aligned}
$$

where $K P I_{E}$ denotes the economic KPI that evaluates the costs of the DWN management, $K P I_{S}$ considers safety KPI, which should be always 0 if the system is running safely. $K P I_{M}$ presents the measured safety KPI that accumulates average volume of remaining water in each storage tank, together with $K P I_{S}$ estimating the safety levels under different settings, and $K P I_{\Delta U}$ addresses smoothness KPI that computes the collected slew rates. Moreover, $n_{s}$ represents the 
number of hours considered in the assessment.

\section{B. Comparison with Certain-equivalent MPC}

As discussed in [23], the certain-equivalent MPC (CE-MPC) for the DWN is formulated by ignoring the propagation of variances of the forecasted water demands, which can be written as the following optimization problem.

\section{Problem 4 (CE-MPC for DWN).}

$$
\min _{\mathbf{u}_{k}^{*}, \xi_{k}^{*}} \sum_{i=0}^{H_{p}-1} \mathcal{L}_{k+i \mid k}
$$

subject to

$$
\begin{aligned}
& \overline{\mathbf{x}}_{k+i+1 \mid k}=\mathbf{A} \overline{\mathbf{x}}_{k+i \mid k}+\widetilde{\mathbf{B}} \widetilde{\mathbf{u}}_{k+i \mid k}+\widetilde{\mathbf{B}}_{p} \overline{\mathbf{d}}_{k+i \mid k} \\
& \mathbf{u}_{\min } \leq \tilde{\mathbf{P}} \tilde{\mathbf{M}}_{1} \tilde{\mathbf{u}}_{k+i \mid k}+\tilde{\mathbf{M}}_{2} \mathbf{d}_{k} \leq \mathbf{u}_{\max } \\
& \mathbf{x}_{\min } \leq \overline{\mathbf{x}}_{k+i+1 \mid k} \leq \mathbf{x}_{\max } \\
& \overline{\mathbf{x}}_{k+i+1 \mid k} \geq \vartheta_{\text {net }, k+i+1 \mid k}-\xi_{k+i \mid k} \geq 0 \\
& \left(\mathbf{x}_{k \mid k}, \mathbf{d}_{k \mid k}\right)=\left(\mathbf{x}_{k}, \mathbf{d}_{k}\right)
\end{aligned}
$$

\section{Results}

All numerical simulations were performed in MATLAB R2014a ${ }^{\circledR}$ 32-bit, running in a PC with CPU of Intel ${ }^{\circledR}$ Core ${ }^{\mathrm{TM}}$ i7-5500U @ $2.40 \mathrm{GHz}$ and RAM of 12GB. The experimental water demand data come from selected consume sectors in the Barcelona DWN. The sampled water demand data are plotted in Figure 4. Weights $\lambda_{1}, \lambda_{2}, \lambda_{3}, \lambda_{4}$ are selected as 100, 10, 1 and 0.1 depending on the prioritization of the operational goals. The common selection for the MPC prediction horizon in a DWN is treated as 24 hours because the water demand cycle is one day.

For purpose of solving the proposed optimization problems with constraints, YALMIP toolbox and Gurobi solver are used, which are able to solve large-scale optimization problems at a satisfied calculation speed in this case study.

By using the DSHW-GP forecasting algorithm, a sequence of disturbance forecasting results are shown in the Figure 5. The validated disturbances (red line) are approximately following the mean estimation (blue line). The gray area include the potential varying of the disturbances.

Simulation results shown in Figure 6 correspond to the tank d110PAP in Figure 3. The top graph in Figure 6 draws the related control actions and the water cost variation with respect to time, where the water flow is increasing as the water cost is decreasing. Meanwhile, the middle graph shows how the water volume in the tank is varying within its limitations and always above the (green) dash line that corresponds with the net demand in terms of the safety constraint setting. Every day, the water volume increases when there are water inflows, and decreases when there are 


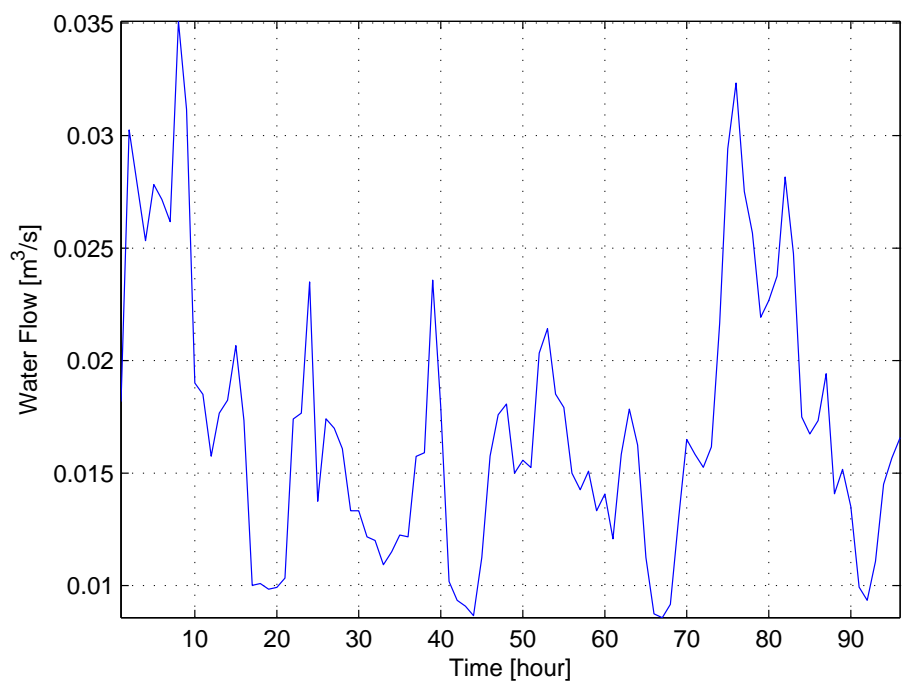

Figure 4. Sampled data of consumer demands

not water inflows and consume demand, which is shown in the bottom plot. In general, the control objectives are properly satisfied and the stochastic demands have little effects on the whole system. The DWN is running under a safe setting.

In order to figure out how the soft term $\eta_{k}$ is varying in the optimization process, Figure 7 shows $\eta_{k}$ for the first system state and the state evolution over $H_{p}$. As discussed above, $\eta_{k}$ is used for avoiding the infeasibility when CI are growing a lot for being useful in the control system. From the plots, the bounds of the state are growing quite fast when $\eta_{k}$ is close to 0 while $\eta_{k}$ is growing when two bounds come close to the physical limitations of the tank. Hence, the critical value for individual risk is slacked successfully. However, as defined in (10b), the sum of all the individual risks would be less than the global risk after risk slacked. But, it is useful to manipulate the unbounded uncertainties.

Table I

Comparisons of Controller Performances

\begin{tabular}{|c|c|c|c|}
\hline Case & $\mathrm{KPI}_{E}$ & $\mathrm{KPI}_{M}$ & Computational Time $[\mathrm{s}]$ \\
\hline CE-MPC & 14.2926 & $8.3014 \times 10^{4}$ & 922.868 \\
\hline GP-MPC @5\% & 14.7538 & $1.3730 \times 10^{5}$ & 924.516 \\
\hline GP-MPC @10\% & 14.7219 & $1.3484 \times 10^{5}$ & 924.449 \\
\hline GP-MPC @15\% & 14.7115 & $1.3375 \times 10^{5}$ & 924.640 \\
\hline GP-MPC @20\% & 14.7097 & $1.3304 \times 10^{5}$ & 924.462 \\
\hline GP-MPC @30\% & 14.7017 & $1.3162 \times 10^{5}$ & 924.516 \\
\hline GP-MPC @50\% & 14.6722 & $1.2989 \times 10^{5}$ & 924.491 \\
\hline
\end{tabular}

Table I compares the results of GP-MPC by using previous four KPIs with different selected risks $\alpha_{\text {global }}$. In these 


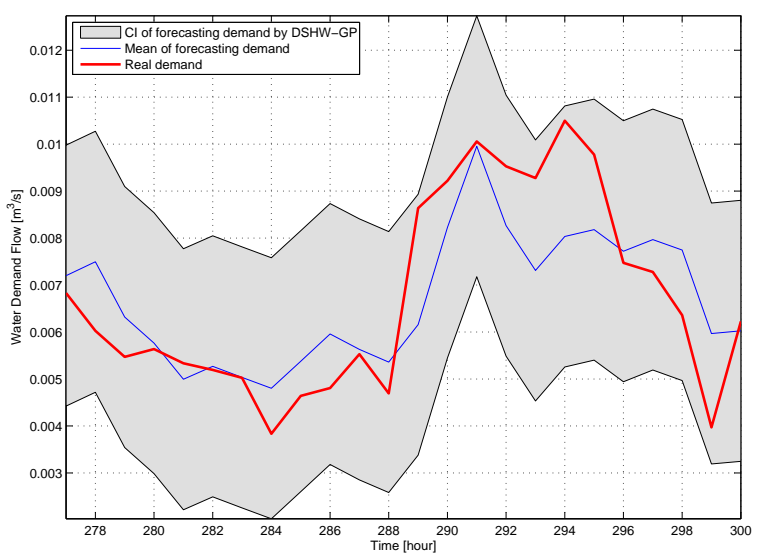

(a) Step 1

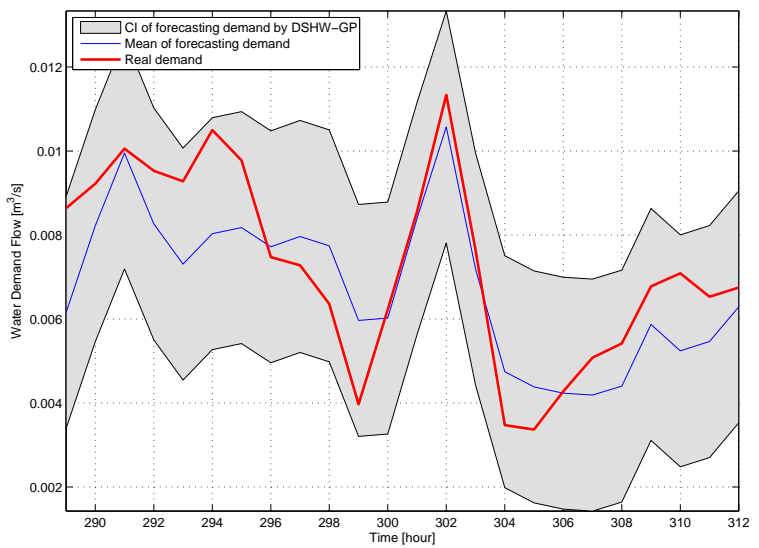

(c) Step 3



(b) Step 2



(d) Step 4

Figure 5. Sequence of forecasting results taken from [14]

simulations, values of $K P I_{S}$ are null in all cases. The values for $K P I_{E}$ should decrease as the risk grows because if the risk is chosen as a small number, more uncertainty information has been propagated to the system state. Consequently, more water is accumulated into the storage tanks in order to guarantee that there is enough water to supply demands. Thus, this process will bring some additional economic costs because of the additional water stocked. Meanwhile, $K P I_{S}$ is null as expected, which means that there is enough water to satisfy stochastic demands along the simulation and $K P I_{M}$ measures the level of obtained safety. If the value of $K P I_{M}$ is larger, the system has more safety to deal with underlying uncertainties. Besides, the results of $K P I_{\Delta U}$ remain the same small value at $4.0806 \times 10^{-6}$, which do not disturb by different risks due to the weights for smoothness cost function are relative small. Furthermore, $K P I_{E}$ of CE-MPC is smaller than the one achieved when using GP-MPC, which means that CE-MPC is economically cheaper than GP-MPC due that $K P I_{E}$ is selected as economic cost in the objective function. $K P I_{M}$ of CE-MPC is also smaller than GP-MPC, which means that, by applying the CE-MPC strategy, brings less safety than GP-MPC because $K P I_{M}$ is calculated by accumulating the part of the water volumes inside storage tanks beyond the net water 

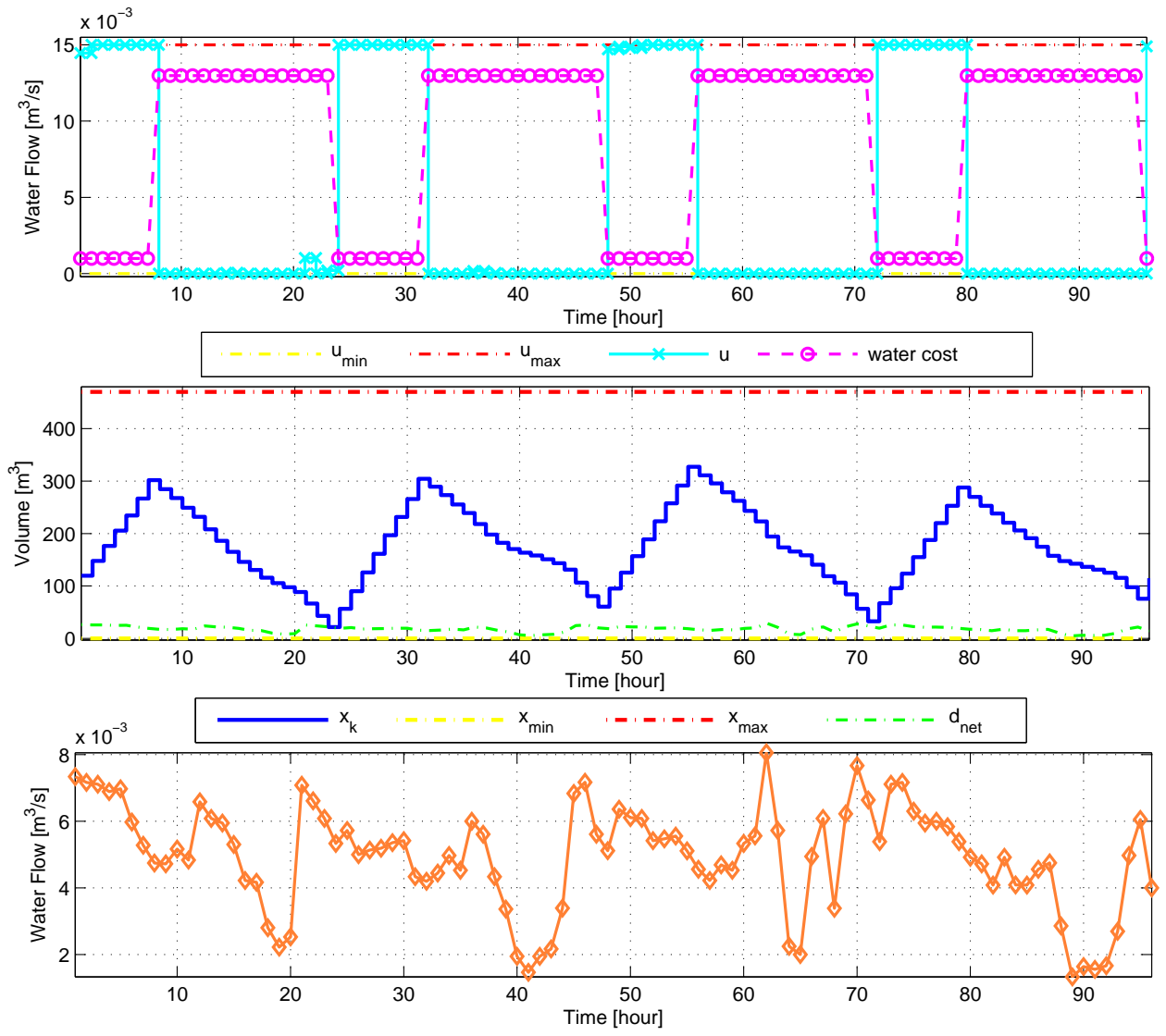

Figure 6. Simulation results for Tank d110PAP

consumptions. Furthermore, $K P I_{S}$ of CE-MPC is still equal to zero, which means that the system is running in a safe way and supplying the required demand.

From Table I, it can be noticed that the computational time is quite similar since the disturbance forecasting consumes most of the total time and therefore the solution time of the optimizations problems behind each considered MPC-based strategy are relatively comparable. Moreover, the computational time in CE-MPC is shorter than GP-MPC because of the reduced complexity of the optimization problem in the former case. Considered time measurements are related to the solution of the optimization problems using YALMIP Toolbox and GUROBI solver.

From the computational times presented in Table I, the proposed GP-MPC strategy can be used to the whole Barcelona DWN since the sampling time used for the GP-MPC is one hour. Moreover, the computational times of the proposed approach is quite similar to the one of CE-MPC already applied to the whole Barcelona network in [23]. In any case, if there would exist computational issues, several approaches might be taken into account. A particular way to circumvent this issue consist in using a partitioning approach based on graph theory, see [24]. This strategy can be used to split the whole system into sybsystems and hence the control in several controllers, which are designed in a hierarchical manner. 

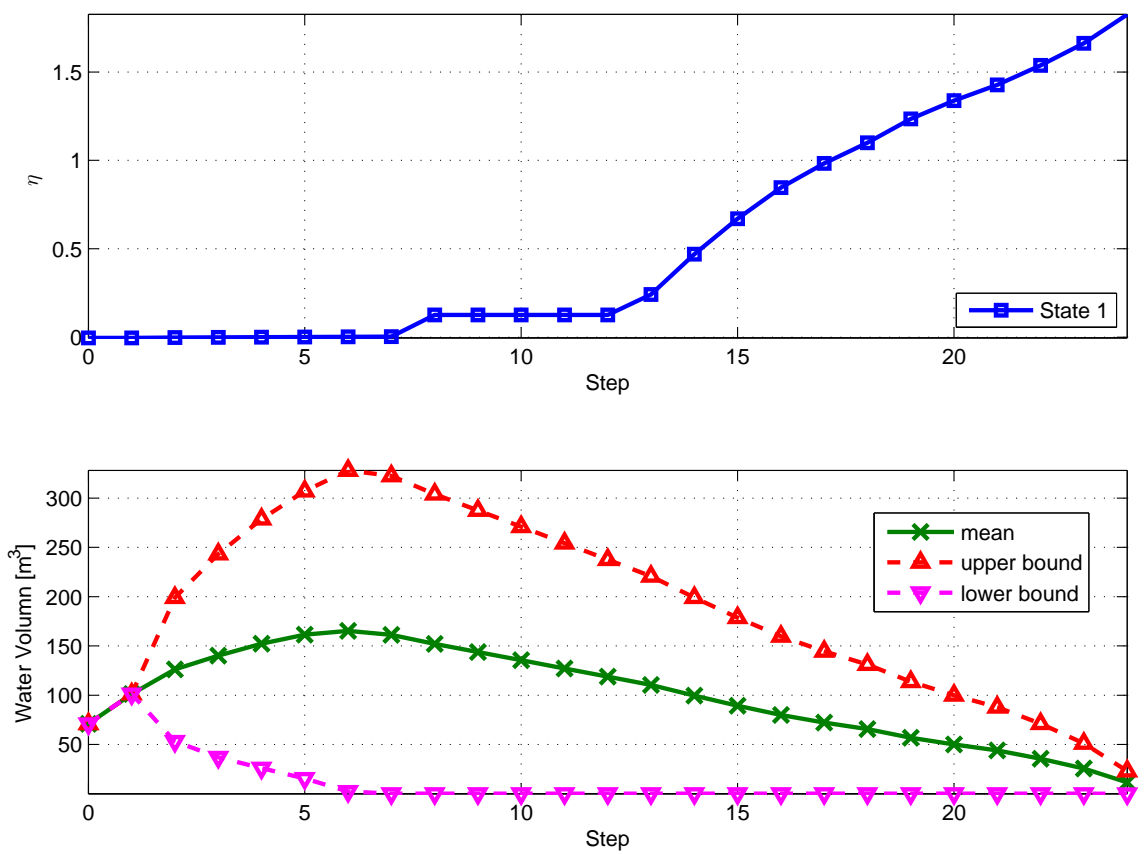

Figure 7. Evolution of $\eta$ for the tank $d 110 P A P$ and water volume evolution along $H_{p}$

\section{CONCLUSIONS}

This paper has developed a new SMPC approach based on GP to manage system disturbances. By using DSHWGP algorithm, the system disturbances have been forecasted over the MPC prediction horizon. The effectiveness and efficiency of applying the proposed approach into the real case have been discussed and analyzed with real demand data gathered from Barcelona DWN. Although dealing with real data involves many difficulties, e.g., the data including a variety of disturbances such as noise and faults of sensors, the proposed GP-MPC has successfully avoided the infeasibility from disturbance measurement by adding a slack variables to propagate system state disturbance over the MPC prediction horizon. The case study of Barcelona DWN has been satisfactorily used to prove that GP-MPC strategy is able to reach a desired performance and maintain the reliability and safety of the whole system.

Future work extending the topics discussed in this paper will be focused on proving robust recursive feasibility and stability of the proposed approach.

\section{ACKNOWLEDGEMENTS}

This work was supported by Spanish Government (Ministerio de Economía y Competitividad) and FEDER under project DPI2014-58104-R (HARCRICS). Moreover, this paper was partially supported by the research project ECOCIS DPI-2013-48243-C2-1-R of the Spanish Ministry of Science and by AGAUR Doctorat Industrial 2013-DI-041. 


\section{REFERENCES}

[1] Campo PJ, Morari M. Robust Model Predictive Control. In: Proceeding of the American Control Conference. Minneapolis, USA; 1987. p. 1021-1026.

[2] Mayne DQ, Seron MM, Rakovic SV. Robust model predictive control of constrained linear systems with bounded disturbances. Automatica. 2005;41(2):219 - 224.

[3] Kocijan J, Murray-Smith R, Rasmussen C, Likar B. Predictive control with Gaussian process models. EUROCON 2003 Computer as a Tool The IEEE Region 8. 2003;1:352-356.

[4] Maciejowski JM, Yang X. Fault tolerant control using Gaussian processes and model predictive control. In: 2013 Conference on Control and Fault-Tolerant Systems (SysTol). Nice, France; 2013. p. 1-12.

[5] Lourenco JM, Santos PJ. Short-term load forecasting using a Gaussian process model: The influence of a derivative term in the input regressor. Intelligent Decision Technologies. 2012;6(4):273-281.

[6] Samarasinghe M, Al-Hawani W. Short-term Forecasting of Electricity Consumption using Gaussian Processes. University of Agder; 2012.

[7] Pawlowski A, Guzman JL, Rodriguez F, Berenguel M, Normey-Rico JE. Predictive Control with Disturbance Forecasting for Greenhouse Diurnal Temperature Control. In: 18th IFAC World Congress. Milano, Italy; 2011. p. 1779-1784.

[8] Fung Y, Tummala VR. Forecasting of electricity consumption: a comparative analysis of regression and artificial neural network models. In: 2nd International Conference on Advances in Power System Control, Operation and Management. vol. 2; 1993. p. 782-787.

[9] Hayati M, Shirvany Y. Artificial Neural Network Approach for Short Term Load Forecasting for Illam Region. World Academy of Science: Engineering and Technology. 2007;22:280-284.

[10] Bakker M, Vreeburg JHG, van Schagen KM, Rietveld LC. A fully adaptive forecasting model for short-term drinking water demand. Environmental Modelling and Software. 2013;48(0):141-151.

[11] Msiza IS, Nelwamondo FV, Marwala T. Water Demand Prediction using Artificial Neural Networks and Support Vector Regression. Digital intelligence. 2008;3(11):1-8.

[12] Rasmussen CE, Williams CKI. Gaussian Processes for Machine Learning. Massachusetts Institute of Technology: the MIT Press; 2006.

[13] Blanch J, Quevedo J, Saludes J, Puig V. Short-term Demand Forecasting For Operational Control of the Barcelona Water Transport Network. In: Conferencia Nacional de Jóvenes Profesionales del Agua de España. Barcelona; 2010. p. 1-10.

[14] Wang Y, Ocampo-Martinez C, Puig V, Quevedo J. Gaussian-process-based demand forecasting for predictive control of drinking water networks. In: 9th International Conference on Critical Information Infrastructures Security. Limassol, Cyprus; 2014. .

[15] Christiaanse WR. Short-Term Load Forecasting Using General Exponential Smoothing. IEEE Transactions on Power Apparatus and Systems. 1971;90(2):900-911.

[16] Harrison PJ. Exponential Smoothing and Short-Term Sales Forecasting. Management Science. 1967;13(11):821-842.

[17] Grosso JM, Ocampo-Martinez C, Puig V, Joseph B. Chance-constrained model predictive control for drinking water networks. Journal of Process Control. 2014;24(5):504-516.

[18] Ono M, Williams B. Iterative Risk Allocation: A New Approach to Robust Model Predictive Control with a Joint Chance Constraint. In: Proceedings of the 47th IEEE Conference on Decision and Control. Cancun, Mexico; 2008. p. 3427-3432.

[19] Scokaert POM, Mayne DQ, Rawlings JB. Suboptimal model predictive control (feasibility implies stability). IEEE Transactions on Automatic Control. 1999;44(3):648-654.

[20] Amrit R, Rawlings JB, Angeli D. Economic optimization using model predictive control with a terminal cost. Annual Reviews in Control. 2011;35(2):178-186.

[21] Angeli D, Amrit R, Rawlings JB. On Average Performance and Stability of Economic Model Predictive Control. IEEE Transactions on Automatic Control. 2012 July;57(7):1615-1626.

[22] Grosso JM, Ocampo-Martinez C, Puig V, Limon D, Pereira M. Economic MPC for the Management of Drinking Water Networks. In: Proceedings of the European Control Conference. Strasburg, France; 2014. p. 1-12. 
[23] Ocampo-Martinez C, Puig V, Cembrano G, Quevedo J. Application of MPC strategies to the management of complex networks of the urban water cycle. IEEE Control Systems Magazine. 2013;33(1):15-41.

[24] Ocampo-Martinez C, Bovo S, Puig V. Partitioning approach oriented to the decentralised predictive control of large-scale systems. Journal of Process Control. 2011;21(5):775 - 786. 